\title{
Structural and characterization studies of insoluble Thai Bombyx mori silk fibroin films
}

\author{
Noor Izyan Syazana Mohd Yusoff a, Mat Uzir Wahit a, ${ }^{\star}$, Juhana Jaafar b, Tuck-Whye Wong b, c \\ a Faculty of Chemical and Energy Engineering, Universiti Teknologi Malaysia, 81310, Johor Malaysia \\ b Advanced Membrane Technology Research Centre (AMTEC), Universiti Teknologi Malaysia, 81310 Skudai, Johor, Malaysia \\ c Faculty of Bioscience and Biomedical Engineering, Universiti Teknologi Malaysia, 81310 Skudai, Johor, Malaysia \\ * Corresponding author: r-uzir@utm.my
}

\section{Article history}

Received 5 May 2018

Revised 1 June 2018

Accepted 2 July 2018

Published Online 4 February 2018

\begin{abstract}
Bombyx Mori fiber consists of two major proteins which are fibroin and sericin. The silk fibroin (SF) is the core structural protein of silk fiber. SF protein structures comprise of primary and secondary structures; where the primary structure contains series of amino acid and secondary structure with Silk I refers to the water-soluble and Silk II, high $\beta$ sheet extent which is insoluble. This study was conducted to compare the structural and characterization of insoluble Thai Bombyx Mori SF with different types of post-treatement. Thai silk cocoons, which were degummed and dissolved in 9.3 $\mathrm{M} \mathrm{LiBr}$ solution at $60{ }^{\circ} \mathrm{C}$. The obtained SF solutions were dialyzed and purified. SF films were prepared by solution casting and immersing in methanol and ethanol, followed by water annealing in water saturated vacuum. Post-treatment was purposely done to regenerate and induce of the $\beta$ sheet structure to enhance the insolubilities and the stabilities properties of the SF films. The SF films structural conformation, characterization and thermal stability were characterized. Attenuated total reflectance-Fourier transformed infrared spectroscopy (ATR-FTIR) showed that SF films were presented in a more stable form after ethanol post treatment, which also supporting by X-ray diffraction (XRD) analysis which indicated the tendency to higher structural organization. Thermal analysis resutls showed that SF was thermally stable and improved after post treatment. The contact angle of post treated SF increased the hydrophobicity of the films. The thai SF films could be the promising candidate for applications in tissue regeneration, optical devices, and flexible electronic displays with the possibility to control the SF structure and properties.
\end{abstract}

Keywords: Silk fibroin films, post-treatment, induce $\beta$ sheet, insoluble films

\section{INTRODUCTION}

Silk as a typical fibrous protein is produced by a variety of insects including silkworm (Bombyx Mori), and spider silk (Nephila Clavipes). Among the native silk proteins, the silkworm silk (Bombyx mori) is the most extensively used due to availability from farming compared to spider silk. Bombyx Mori has been used as high-quality textile fiber and suture for a long time. Silk fibroin (SF) is the core structural protein of silk fiber and can be used in various forms, such as gels, powders, fibers, or membranes, depending on application besides of its utility as a textile fiber (Asakura et al., 1985; Li et al., 2002; Putthanarat et al., 2002). Many researchers have recently investigated SF as one of candidate materials for biomedical applications since it has several useful properties including good biocompatibility, good oxygen and water vapor permeability, biodegradability, non-toxic, and minimal inflammatory reaction (Jin et al., 2004; Yoon et al., 2014; Lee et al., 2016).

The preparation of the regenerated Thai SF generally involves pretreatments of the silk cocoons by degumming, dissolving the SF fiber in salt solution, dialysis to remove the sovent and fabrication through solution casting. The post-treatments were performed for regenerating the protein's secondary structures. These multi-step processes have been shown to affect molecular weights and structures of the SF (Cho et al., 2012; Aznar-Cervantes et al., 2016). SF structure consists of the combination of the secondary structures with the Silk I refers to the water-soluble and Silk II, high $\beta$ sheet extent which is insoluble (Sashina et al., 2006).

SF films prepared by solution casting at room temperature without any post-treatment are soluble in water and brittle at room tempertaure. These properties can possibly be detrimental to many applications due to the dominating random-coil structures. Hence, important parameters of SF films that should be controlled for biomaterial application are the molecular conformation of SF and the elasticity and malleability of the films (Silva et al., 2013). Therefore, by introducing post-treatment using solvents or water annealing, the modification of the secondary structure of SF is possible.

The purpose of post-treatment by methanol immersion, ethanol immersion and water vapor annealing is to regenerate construction of the $\beta$ sheet structure in order to improve the insolubilities and the stabilities properties of SF films (Lu et al., 2010; Seib et al., 2012). The study of structural changes of Thai SF films by these methods; immersion in organic solvents and water annealing is a very interesting study to be explored in fundamental and applied researches for enhancement of this biopolymer for new applications.

Previous studies have been shown that post treated SF films with methanol exhibited the increase in $\beta$-sheet content when comparing to water-annealed silk films (Lawrence et al., 2008). Study by Nogueira in using of ethanol treatment, showed that membranes were crystallized to the $\beta$ sheet conformation (Nogueira et al., 2010). Recent study showed that, water annealing treatment would induce the formation of silk I and silk II in SF films (Jaramillo-Quiceno et al., 
2017). For that reason, it is important to identify and understand how the Thai silk films properties respond to the post treatment. By better comprehending on how post treatment influences the properties of Thai silk films, variable processing methods can be customized based on the application.

The Thailand silkworms having yellow cocoons which are different from Chinese and Japanese cocoons which are white in colour. It attracts our interest to study the structural and characteristics of silk fibroin from Thai Bombyx Mori since most of the previous studies are is based on Japanese (Hu et al., 2006; Lu et al., 2010; Zhang et al., 2012) and China (Hu et al., 2012; Huang et al., 2014) cocoons and only few of them are using the same Thai cocoons (Chankow et al., 2016) for the structural studies. The goal of this study wasis to characterize the structural properties of SF films and the effect of different types of post-treatment of the SF films from Thai Bombyx Mori, by aiming to form an insoluble SF film with a more stable and crystalline structure. No systematically report from previous research has investigated the characterization and structural properties of Thai silk fibroin film after posttreatment with different types of solvent/method.

Based on that, this study would report on structural and characterization of insoluble Thai Bombyx Mori silk fibroin films through solution casting method. In this study, we aimed to relate the understanding of the SF films characterization and structural through ATR-FTIR, XRD, and UV-vis. We also evaluated the influences of post-treatment on the wettability, thermal stability and the morphology of the silk films.

\section{EXPERIMENTAL}

\section{Materials}

Bombyx Mori cocoon was purchased from The Queen Sirikit Sericulture Centre Surin, Thailand. Sodium carbonate $\left(\mathrm{Na}_{2} \mathrm{CO}_{3}\right)$, lithium bromide $(\mathrm{LiBr})$, ethanol $(\mathrm{EtOH})$ and methanol $(\mathrm{MeOH})$ were all analytical grade chemicals.

\section{Preparation of the silk fibroin solution}

Cocoons of the Thai Bombyx Mori silkworms were cut into 4 or 5 pieces until it reached $5 \mathrm{~g}$ of silk cocoons for one batch of silk fibroin extraction. The silk fibroin was immersed in $0.02 \mathrm{M} \mathrm{Na}_{2} \mathrm{CO}_{3}$ at $100^{\circ} \mathrm{C}$ for $30 \mathrm{~min}$ to remove the sericin proteins. Then raw silk fibroin was rinsed thoroughly with water for 20 minutes in 3 times and left to dry at room temperature for 2 days. The extracted SF was dissolved in 9.3 $\mathrm{M} \mathrm{LiBr}$ for $4 \mathrm{~h}$ at $60{ }^{\circ} \mathrm{C}$ to generate a $20 \% \mathrm{w} / \mathrm{v}$ solution. Then the solution was dialyzed using $3.5 \mathrm{kDa}$ dialysis tubing (Sigma Aldrich) against distilled water for 48 hours with 8 total water changes. The silk fibroin solution was centrifuged at $900 \mathrm{rpm}, 4^{\circ} \mathrm{C}$ for 20 minutes to remove impurities available in solution. The resultant of $6 \% \mathrm{w} / \mathrm{v}$, determined by weighing the remaining solid was left in oven at $60^{\circ} \mathrm{C}$ until it was completely dried. The SF dissolution was stored at $4{ }^{\circ} \mathrm{C}$ for not longer than 30 days.

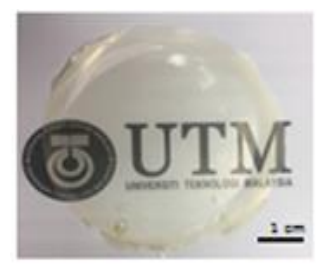

Silk fibroin film

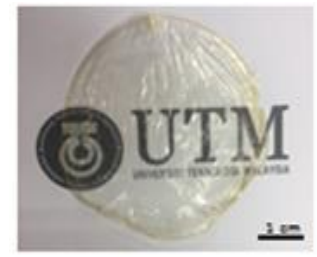

Silk fibroin_methanol film

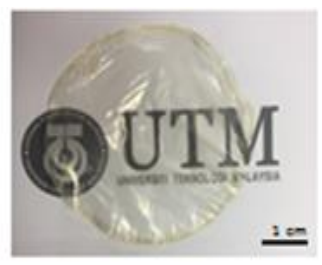

Silk fibroin ethanol film

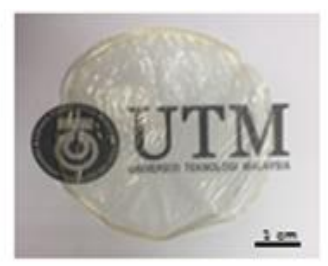

Silk fibroin_water anneling film
Fig. 1 Image of silk fibroin films.

\section{Fabrication of the silk fibroin films}

The silk fibroin films were prepared by pouring them into the Teflon mould. The silk fibroin composite films were obtained after leaving them to dry for 24 hours at ambient temperature. One set of SF films was immersed in methanol and ethanol $(80: 20 \% \mathrm{v} / \mathrm{v})$ for an 1 hour to induce $\beta$-sheet formation. After being submerged in alcohol solution, the SF samples were left to dry for 24 hours in room temperature. Another one set of SF films was treated with waterannealing processing by placing the films on the teflon mold on a shelf in a $24 \mathrm{mmHg}$ vacuum oven filled with water bath for 6 hour to create saturated water vapor environment that would induce the formation of $\beta$-sheet secondary structure within the silk film. The films were left to dry. The thickness of the films was measured with a digital micrometer in the range of $80-90 \mu \mathrm{m}$. The dried films were stored in a moisture controlled desiccator for further testing.

\section{Structural study of SF films}

Attenuated total reflectance-Fourier transform infrared (ATRFTIR) spectra were recorded using Shiamdzu (Japan) spectrometer at ambient temperature. The spectra were taken at $4.0 \mathrm{~cm}^{-1}$ resolution. 64 scans were accumulated to get a reasonable signal to noise ratio. The characterization of the chemical structures of SF films was identified using ATR-FTIR. Conformational transitions between random coils and alpha helices in silk I and silk II consisted of beta sheets/ beta turns structures of the Thai SF (Hu et al., 2006; Chankow et al., 2016).

$\mathrm{X}$-ray diffraction (XRD) results were obtained using Rigaku Miniflex II with $\mathrm{Cu} \mathrm{K} \alpha$ radiation (wavelength $=1.54 \AA$ ) at $40 \mathrm{kV}$ and $30 \mathrm{~mA}$. The results of XRD were recorded in the region of $2 \theta$ from $5^{\circ}$ to $70^{\circ}$. XRD has been used to recognize the crystalline region of SF films before and after post-treatment, which would influence the physicochemical properties of the majority of natural polymer (Tsukada et al., 1994).

Bragg's law was applied to identify the interplanar distances of different peaks:

$$
\text { Interplanar distance }=\mathrm{n} \lambda /(2 \sin \theta)
$$

where; $\mathrm{n}$ is the order of diffraction $(\mathrm{n}=1) ; \lambda$ is the $\mathrm{X}$-ray wavelength and $\theta$ is Bragg's angle.

Ultraviolet-visible (UV-vis) spectroscopy was applied to identify the optical transmittances of the SF films. The range used was from 200 to $800 \mathrm{~nm}$ at room temperature by using Shimadzu UV-3101PC, Japan. UV-vis was used for investigating the optical behavior of the SF films which have smooth surfaces, highly transparent across the visible region and possess high refractive index (Asha et al., 2015).

\section{Wettability study}

Wettability studies of SF and SF post-treatment films were determined using water contact angle measurement using the sessile drop method with an optical bench type contact angle goniometer (Kruss Gambult, Germany). For each film samples, at least three measurements were taken. The average value of contact angle was obtained.

\section{Thermal stability}

The thermal stability of the silk fibroin films was analyzed by thermal gravimetric analysis (TGA) using TA Q600 instrument. This process was conducted in nitrogen $\left(\mathrm{N}_{2}\right)$ ambient, under the heating rate of $10{ }^{\circ} \mathrm{C} \mathrm{min}-1$ from $30{ }^{\circ} \mathrm{C}$ to $600{ }^{\circ} \mathrm{C}$.

\section{Morphology analysis}

The morphology analysis of the SF films and post treated SF films was analyzed using field emission scanning electron microscopy (FESEM: Zeiss Supra 35VP). The samples were air dried and kept in a dessicator. Samples were finally subjected to gold sputtering and scanned under FESEM. 


\section{RESULTS AND DISCUSSION}

\section{Sturctural Characteristic of Silk Fibroin Films}

Structural analyses of the SF films were first determined by ATRFTIR. The absorbance spectra of silk fibroin film and after posttreatment were shown in Fig.2 (a). The IR spectral region within 600 $4000 \mathrm{~cm}^{-1}$ was performed to identify chemical structure of SF films. The absorption of the peptide backbones in region $1700-1600 \mathrm{~cm}^{-1}$ for amide I and $1600-1500 \mathrm{~cm}^{-1}$ of Amide II. These two main region are commonly used for the analysis of the secondary structure of the silk fibroin.The observed peaks at $1610-1630 \mathrm{~cm}^{-1}$ (amide I) and $1510-1520 \mathrm{~cm}^{-1}$ (amide II) were characteristic of silk II secondary structure, whereas the absorptions at $1640-1654 \mathrm{~cm}^{-1}$ (amide I) and $1535-1545 \mathrm{~cm}^{-1}$ (amide II) were indicative of silk I structure. The peaks around $1230-1250 \mathrm{~cm}^{-1}$ were referred to amide III $(\mathrm{N}-\mathrm{H}$ deformation, $\mathrm{C}-\mathrm{N}$ stretching), which attributed to random coil and $\beta$ sheet conformation, respectively (Jin et al., 2005; Hu et al., 2006; Lu et al., 2010; Zhang et al., 2012). The amide peaks were commonly found in the protein structure such as silk fibroin.

(a)

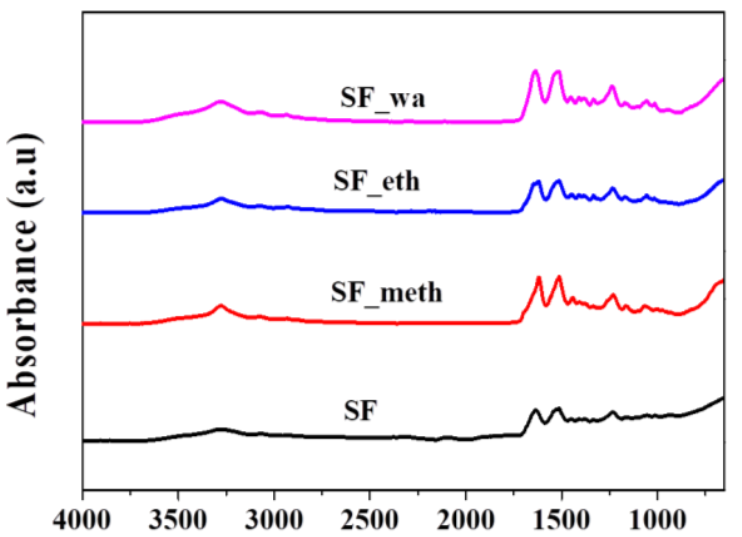

Wavenumber $\left(\mathrm{cm}^{-1}\right)$

(b)

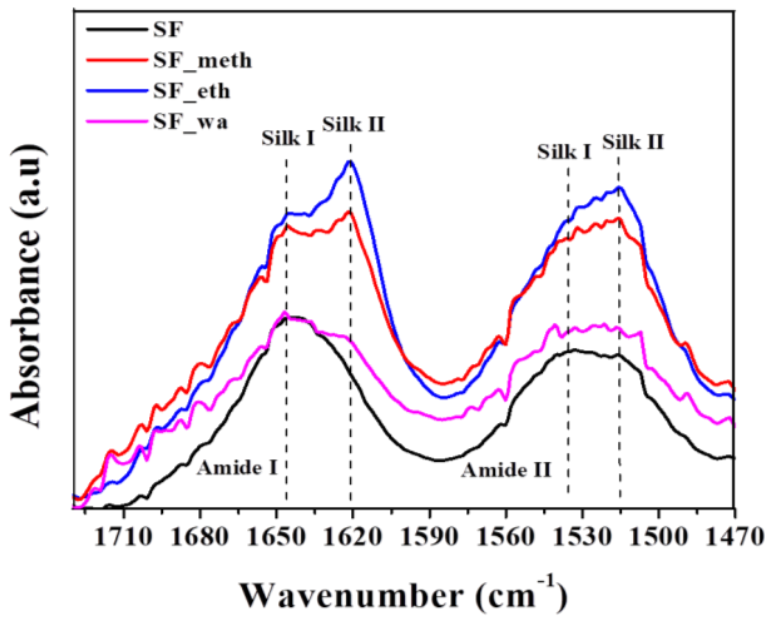

Fig. 2 . ATR-FTIR spectra of SF film, SF_methanol film, SF_ethanol film and SF_water annealing film: (a) in range of $4000 \mathrm{~cm}^{-1}$ to $600 \mathrm{~cm}^{-1}$ (b) Amide I and II peak.

In Fig. 2(b), it was revealed that high absorption intensity at the peak position $1640 \mathrm{~cm}^{-1}$ was corresponded to the $\beta$ sheet conformation of amide I ( $\mathrm{C}=\mathrm{O}$ stretching). A peak was found around $1520 \mathrm{~cm}^{-1}$ attributed to the $\beta$ sheet conformation of amide II ( $\mathrm{N}-\mathrm{H}$ bending, $\mathrm{C}-\mathrm{N}$ stretching) for SF film. After the post-treatment with water annealing, the film showed similar peak with SF film however it was more broadening peak. The peaks for Amide I and II of the methanol and ethanol treated films were shifted to $1620 \mathrm{~cm}^{-1}$ and $1510 \mathrm{~cm}^{-1}$. The silk II structure was generated from the post-treatment process. The results of the SF_ethanol films indicated that the peaks were shifted and broaden more compared to the SF_methanol and SF_water annealing films, indicating more regular silk II structure was formed.
Secondary structures of SF film and post-treatment SF films were further analyzed by X-ray diffraction technique. XRD results of the silk fibroin films were shown in the Figure 3. The SF films showed an XRD pattern of typical amorphous material with the absence of crystallinity peaks. This result was expected as no post-treatment was performed to increase the crystallinity of the films.

The silk films crystallinity was characterized by using XRD at room temperature. The XRD spectra were shown in Figure 3. The pure silk fibroin films exhibited an amorphous structure since no sharp peak wasare displayed. The post-treatment by using methanol immersion, ethanol immersion and water annealing, exhibited new crystallization peaks at $11.7^{\circ}, 20.2^{\circ}, 24.5^{\circ}$ and $36.7^{\circ}$; indicating that fibroin primarily formed crystalline structure. For SF, previous study revealed the main diffraction peaks of the silk I structure were at $12.2^{\circ}, 19.7^{\circ}, 24.7^{\circ}$ and $28.2^{\circ}$, while those of the silk II structure were at $9.1^{\circ}, 18.9^{\circ}, 20.7^{\circ}$ and $24.3^{\circ}$ (Wang et al., 2014).

From the XRD scans, it could be seen that there were crystalline halos for the post-treatment SF film by methanol, ethanol and water annealing compared to silk fibroin film. The diffraction peaks at $2 \theta=$ $11.7^{\circ}, 20.2^{\circ}$, and $36.7^{\circ}$ indicated that post-treatment of the SF induced fibroin to transform from random coils to Silk I. The peak arose at $20.2^{\circ}$ was corresponded to the $\mathrm{d}$-spacing of $4.4 \AA$ for silk II structure. The changes in crystallinity of the film wer due to increase in $\beta$-sheet crystalline structure of fibroin after the post-treatment(Abdel-Fattah et $a l ., 2015)$.The results from XRD were corroborated with FTIR results, which further confirmed that the post-treatment was affected the crystallinity of the films. A crystallisation peak appeared at $24.7^{\circ}$ was indicated that the post-treatment of SF film also contained Silk II crystalline structure eventhough the peak was not visible for SF neat.

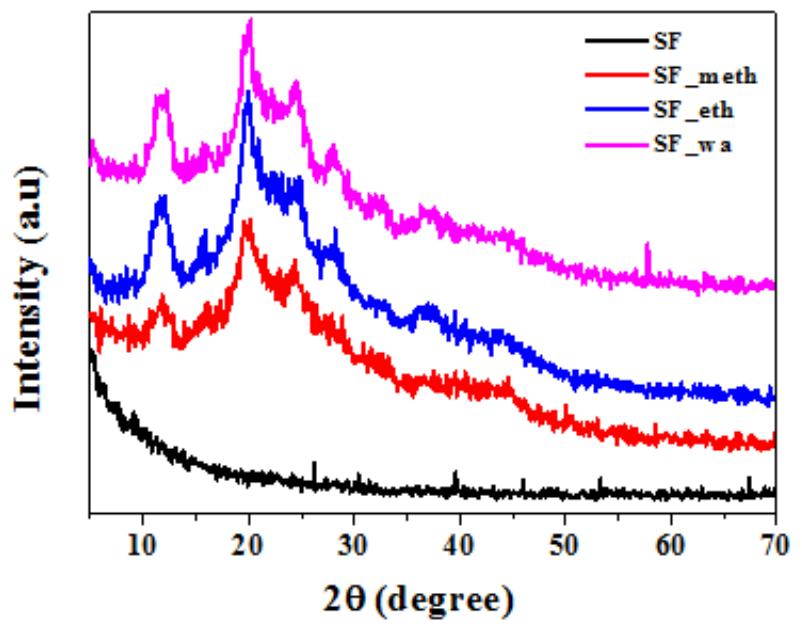

Fig. 3 . XRD spectra of SF film, SF_methanol film, SF_ethanol and SF water annealing film.

Fig. 4 compares the UV-vis spectroscopies of SF film and posttreatment SF films. All the films showed similar transmittance values. The films were strongly absorbed in the UV region of the spectrum (below $350 \mathrm{~nm}$ ). Strong bands at $250 \mathrm{~nm}$ were characteristic for SF film, SF_methanol, SF_ethanol and SF_water annealing, respectively, which attributed to the $\pi$ - $\pi *$ electron transition of the amino acid residues in protein chain of silk fibroin (Autran et al., 2010). To compare the transparency of the SF films and the post-treatment films, the transmittance at $550 \mathrm{~nm}$ was chosen based on the wavelengths where human eyes are highly sensitive (Liu et al., 2012). All films showed above $70 \%$ transparency across the visible light spectrum (400 $-800 \mathrm{~nm}$ ), reflecting that all the films were transparence.

The SF films were comparatively amorphous than the post treated SF films due to the fact that SF films have the closest transmittance value to poly(methyl methacrylate) (PMMA), a polymer which is highly amorphous with around 75\% transparency (Lee et al., 2016). Therefore, the transparency in SF films reflected the structure of polymer chains in which they were amorphous or appreciable 
crystalline. This proven that by post-treatment of the SF films has transformed to crystalline region from amorphous region.

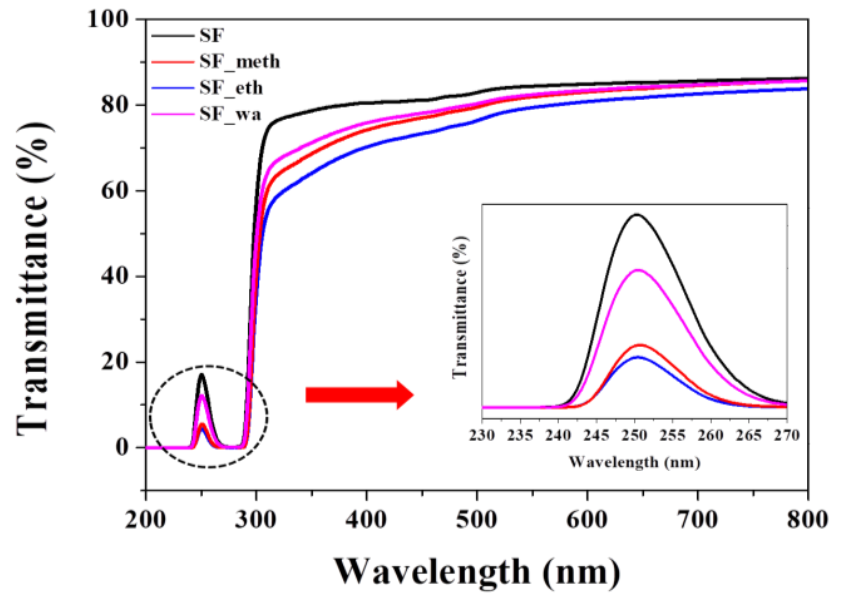

Fig. 4 UV-vis spectra of SF film, SF_methanol film, SF_ethanol film and SF_water annealing film.

\section{Thermal stability}

The TGA and DTG curves of the SF films were shown in Fig. 5. The SF films before and after post treated films showed a mass loss of about $8 \%$ at $100{ }^{\circ} \mathrm{C}$ due to the removal of the water content in the films. The thermal degradation of the SF films was occurred at about $251{ }^{\circ} \mathrm{C}$. After the post-treatment, all the SF films yielded similar TGA curves. However, there was obvious contrast between the pure SF film and the post-treatmnet SF films. The degradation of silk fibroin films at around 254-270 ${ }^{\circ} \mathrm{C}$ (Table 1) was associated with the breakdown of side chain groups of amino acid residues as well as the cleavage of peptide bonds (Um et al., 2001; Nogueira et al., 2010). The results summarized that, SF_ethanol film has higher degradation compared to SF_methanol, SF_water annealing and SF films, which was related to the increase of $\beta$-sheet secondary structure formation within the film bulk material of ethanol treated SF film. This result has been discussed in the literature previously (Hu et al., 2006; Lawrence et al., 2010).

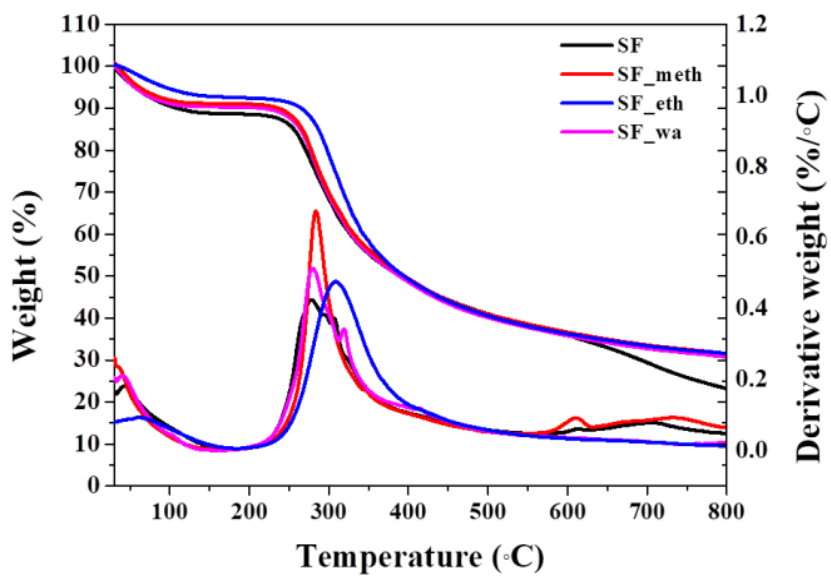

Fig. 5 TGA and DTG curves of silk fibroin films.

Table 1 Thermal degradation of the silk fibroin films.

\begin{tabular}{cc}
\hline Sample & Temperature $\left({ }^{\circ} \mathrm{C}\right)$ \\
\hline SF & 251 \\
SF_methanol & 256 \\
SF_ethanol & 270 \\
SF_water annealing & 254
\end{tabular}

Furthermore, the total weight loss of the SF film was the highest compared to the post treated SF films at $800{ }^{\circ} \mathrm{C}$, which was at $79 \%$. As expected, post-treament of silk fibroin film would stabilize the films at high temperature. Moreover, the higher content of silk II structure would offer synergistic effects to improve the thermal stability of the SF films.

Water contact angle measurements on the SF films and post treated SF films were shown in Table 2. The average measurement of SF films was the lowest compared to the post treated SF films. After the post-treatmemt, an increment could be seen at the contact angle measurement due to the increase in $\beta$-sheet where the films became more hydrophobic and formed insoluble films. The observation demonstrated that post treated silk fibroin has significant influence on its hydrophobicity due to changes from random coil to $\beta$-sheet conformation. Higher contact angle of SF_ethanol was observed due to higher $\beta$ sheet content compared to others. These trends might be attributed to the effect of increased surface roughness as supported from FESEM results.

Table 2 Contact angle measurements of SF films $(n=3$, average \pm SD).

Sample
$\begin{gathered}\text { Equilibrium contact } \\ \text { angle }\left(^{\circ}\right) \pm \text { SD }\end{gathered}$
SF
SF_ethanol
SF_methanol
SF_water anealling
The cross-section and surface morphology of the SF film and post
treated SF_ethanol films were recorded using FESEM (Fig. 6). A
similar characteristic was discovered from the surface images of the SF
film and SF_ethanol; both were relatively homogenous and rough
However, SF_ethanol films were more roughcompared to SF films.
Noticeable difference was observed in the cross-section of the two sets
of films, where the cross sectional area of SF film was smoother
compared to SF_ethanol film. Thus, it could be concluded that post
treated SF with ethanol would cause irregularity of the film surface and
cross section due to the dehydrating solvent of ethanol that promoted
chains transformation.
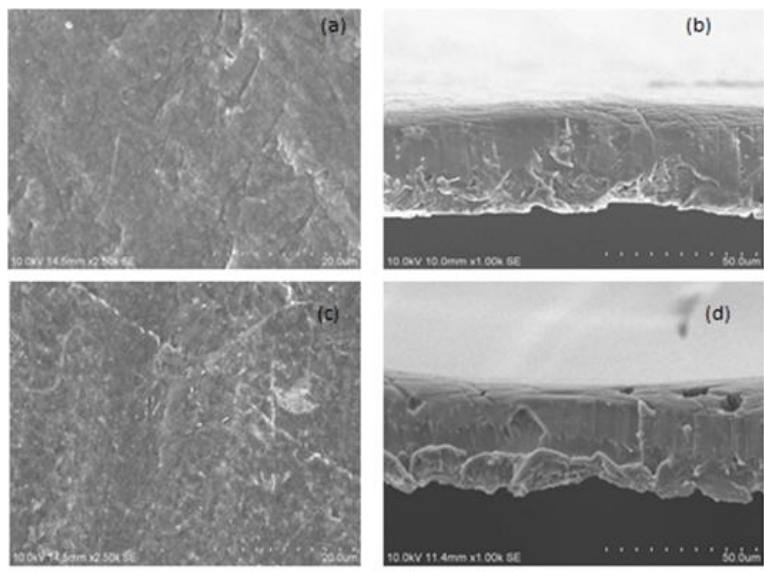

Fig. 6 FESEM images of the SF film, surface (a) and cross-section (b); and the SF_ethanol, surface (c) and cross-section (d). 


\section{CONCLUSION}

The effects on structural and characterization properties of the Thai silk fibroin before and after post-treatment have been studied. The ATR-FTIR,XRD and UV-vis results indicated that the predominance of silk II structure was occurred after the post-treatment by methanol, ethanol and water annealing. The transparencies of the Thai silk fibroin films were not affected by the post-treatment of the sample. The thermal stabillity of the silk fibroin films after post-treatment showed better stability especially for the ethanol treatment. The wettability properties of the silk fibroins film showed better hydrophobicity and insolubility properties of the silk fibroin films. As a results, the Thai silk films after post-treatment via ethanol demostrated more stable silk fibroin film. The thai silk fibroin film could be the promising candidate for the applications in tissue regeneration, optical devices, and flexible electronic displays.

\section{ACKNOWLEDGEMENT}

We would like to thanks Chulalangkorn University for providing the Bombyx Mori Cocoons.This reserch was financially supported under the Research University Grant (GUP:18H85),Universiti Teknologi Malaysia and Ministry of Higher Education Malaysia (MyBrain15).

\section{REFERENCES}

Abdel-Fattah, W. I., Atwa, N., Ali, G. W. (2015). Influence of the protocol of fibroin extraction on the antibiotic activities of the constructed composites. Progress in Biomaterials, 4(2), 77-88.

Asakura, T., Kuzuhara, A., Tabeta, R., Saito, H. (1985). Conformational characterization of Bombyx mori silk fibroin in the solid state by high-frequency carbon-13 cross polarization-magic angle spinning NMR $\mathrm{x}$-ray diffraction, and infrared spectroscopy. Macromolecules, 18(10), $1841-1845$

Asha, S., Sangappa, Y., Ganesh, S. (2015). Tuning the refractive index and optical band gap of silk fibroin films by electron irradiation. Journal of Spectroscopy, 7.

Autran, P., Xavier, C., Dulce, G., Eduardo, L. (2010). Follow up of a model used for extraction fibroin by UV-VIS, XRD, FTIR and SEM/EDS. In: Congress of artificial organs and biomaterials.

Aznar-Cervantes, S., Martínez, J. G., Bernabeu-Esclapez, A., Lozano-Pérez, A A., Meseguer-Olmo, L., Otero, T. F., et al. (2016). Fabrication of electrospun silk fibroin scaffolds coated with graphene oxide and reduced graphene for applications in biomedicine. Bioelectrochemistry, 108, 36-45.

Chankow, S., Luemunkong, S., Kanokpanont, S. (2016, 7-9 Dec. 2016) Conformational transitions of thai silk fibroin secondary structures. Paper presented at the 2016 9th Biomedical Engineering International Conference (BMEiCON), 1-5.

Cho, H. J., Ki, C. S., Oh, H., Lee, K. H., Um, I. C. (2012). Molecular weight distribution and solution properties of silk fibroins with different dissolution conditions. International Journal of Biological Macromolecules, 51(3), 336-341.

Hu, Y., Zhang, Q., You, R., Wang, L., Li, M. (2012). The relationship between secondary structure and biodegradation behavior of silk fibroin scaffolds. Advances in Materials Science and Engineering, 2012, 5.

Huang, X., Fan, S., Altayp, A. I. M., Zhang, Y., Shao, H., Hu, X., et al. (2014) Tunable structures and properties of electrospun regenerated silk fibroin mats annealed in water vapor at different times and temperatures. Journal of Nanomaterials, 2014, 7.

$\mathrm{Hu}$, X., Kaplan, D., and Cebe, P. (2006). Determining beta-sheet crystallinity in fibrous proteins by thermal analysis and infrared spectroscopy. Macromolecules, 39(18), 6161-6170.
Jaramillo-Quiceno, N., Álvarez-López, C., Restrepo-Osorio, A. (2017). Structural and thermal properties of silk fibroin films obtained from cocoon and waste silk fibers as raw materials. Procedia Engineering, 200(Supplement C), 384-388.

Jin, H.-J., Park, J., Valluzzi, R., Cebe, P., Kaplan, D. L. (2004). Biomaterial films of bombyx mori silk fibroin with poly(ethylene oxide). Biomacromolecules, 5(3), 711-717.

Jin, H. J., Park, J., Karageorgiou, V., Kim, U. J., Valluzzi, R., Cebe, P., et al. (2005). Water-stable silk films with reduced $\beta$-sheet content. Advanced Functional Materials, 15(8), 1241-1247.

Lawrence, B. D., Omenetto, F., Chui, K., Kaplan, D. L. (2008). Processing methods to control silk fibroin film biomaterial features. Journal of Materials Science, 43(21), 6967.

Lawrence, B. D., Wharram, S., Kluge, J. A., Leisk, G. G., Omenetto, F. G., Rosenblatt, M. I., et al. (2010). Effect of hydration on silk film material properties. Macromolecular Bioscience, 10(4), 393-403.

Lee, M. C., Kim, D.-K., Lee, O. J., Kim, J.-H., Ju, H. W., Lee, J. M., et al. (2016). Fabrication of silk fibroin film using centrifugal casting technique for corneal tissue engineering. Journal of Biomedical Materials Research Part B: Applied Biomaterials, 104(3), 508-514.

Lee, X., Wahit, M. U., Adrus, N. (2016). Biodegradable and temperatureresponsive thermoset polyesters with renewable monomers. Journal of Applied Polymer Science, 133(40), 1-9.

Li, M., Lu, S., Wu, Z., Tan, K., Minoura, N., Kuga, S. (2002). Structure and properties of silk fibroin-poly(vinyl alcohol) gel. International Journal of Biological Macromolecules, 30(2), 89-94.

Liu, M., Zhang, Y., Wu, C., Xiong, S., Zhou, C. (2012). Chitosan/halloysite nanotubes bionanocomposites: Structure, mechanical properties and biocompatibility. International Journal of Biological Macromolecules, 51(4), 566-575.

Lu, Q., Hu, X., Wang, X., Kluge, J. A., Lu, S., Cebe, P., et al. (2010). Waterinsoluble silk films with silk I structure. Acta biomaterialia, 6(4), 13801387.

Nogueira, G. M., Rodas, A. C. D., Leite, C. A. P., Giles, C., Higa, O. Z., Polakiewicz, B., et al. (2010). Preparation and characterization of ethanoltreated silk fibroin dense membranes for biomaterials application using waste silk fibers as raw material. Bioresource Technology, 101(21), 84468451.

Putthanarat, S., Zarkoob, S., Magoshi, J., Chen, J. A., Eby, R. K., Stone, M., et al. (2002). Effect of processing temperature on the morphology of silk membranes. Polymer, 43(12), 3405-3413.

Sashina, E. S., Bochek, A. M., Novoselov, N. P., Kirichenko, D. A. (2006). Structure and solubility of natural silk fibroin. Russian Journal of Applied Chemistry, 79(6), 869-876.

Seib, F. P., Maitz, M. F., Hu, X., Werner, C., Kaplan, D. L. (2012). Impact of processing parameters on the haemocompatibility of Bombyx mori silk films. Biomaterials, 33(4), 1017-1023.

Silva, M. F., de Moraes, M. A., Nogueira, G. M., Rodas, A. C. D., Higa, O. Z., Beppu, M. M. (2013). Glycerin and ethanol as additives on silk fibroin films: Insoluble and malleable films. Journal of Applied Polymer Science, 128(1), 115-122.

Tsukada, M., Gotoh, Y., Nagura, M., Minoura, N., Kasai, N., Freddi, G. (1994). Structural changes of silk fibroin membranes induced by immersion in methanol aqueous solutions. Journal of Polymer Science Part B: Polymer Physics, 32(5), 961-968.

Um, I. C., Kweon, H., Park, Y. H., Hudson, S. (2001). Structural characteristics and properties of the regenerated silk fibroin prepared from formic acid. International Journal of Biological Macromolecules, 29(2), 91-97.

Wang, L., Lu, C., Zhang, B., Zhao, B., Wu, F., Guan, S. (2014). Fabrication and characterization of flexible silk fibroin films reinforced with graphene oxide for biomedical applications. RSC Advances, 4(76), 40312-40320.

Yoon, H., Kim, E. Y., Kim, H., Park, C. H., Joo, C.-K., Khang, G. (2014). Fabrication of transparent silk fibroin film for the regeneration of corneal endothelial cells; preliminary study. Macromolecular Research, 22(3), 297-303.

Zhang, C., Song, D., Lu, Q., Hu, X., Kaplan, D. L., Zhu, H. (2012). Flexibility regeneration of silk fibroin in vitro. Biomacromolecules, 13(7), 2148-2153. 